\title{
DOSE RATE EFFECTS IN WLS FIBERS
}

\author{
M. David, A. Gomes, A. Maio \\ LIP Av. Elias Garcia $141^{0}, 1000$ Lisbon, Portugal, and FCUL
}

\begin{abstract}
An experiment of low dose rate irradiation of WLS fibers was performed. WLS fibers from Bicron, Kuraray and Pol.Hi.Tech doped with and without UltraViolet absorber (UVA), were irradiated in a ${ }^{60} \mathrm{Co} \gamma$ source. A total dose of $\sim 140 \mathrm{Krad}$ was applied. 3 dose rates were used: $0.55 \mathrm{Krad} / \mathrm{h}, 1.1 \mathrm{Krad} / \mathrm{h}$ and $4 \mathrm{Krad} / \mathrm{h}$. No low dose rate effects were observed.

BCF91A and Y11(200) fibers with single cladding and multicladding, were irradiated in a mixed field of $20 \%$ of neutrons and $80 \%$ of $\gamma$ 's. A total dose of $1 \mathrm{Mrad}$ was applied at a peak dose rate of $1.5 \mathrm{Mrad} / \mathrm{h}$. The radiation effect on single cladding vs. multicladding fibers is compared, and are similar. The high dose rate irradiation effect on fibers is compatible with the much lower dose rate irradiation effect.
\end{abstract}

\section{Introduction}

TILECAL is the sampling hadronic calorimeter to be used in the barrel and extended barrel region of the ATLAS detector. It uses iron as passive material, and scintillating tiles as active material. The tiles are readout by WLS which lead the signal to the PMT's, [1] [2].

The high luminosity $\left(\mathcal{L}=10^{34} \mathrm{~cm}^{-2} \mathrm{~s}^{-1}\right)$ at which the detectors have to operate, imposes stringent requirements in the detector components concerning radiation damage. High dose levels of ionizing radiation are expected, especially in the inner detectors and calorimeters, as shown in the isodose map of fig. 1 obtained by Monte-Carlo simulation [3].

In the TILECAL calorimeter, the sensitive components are the scintillator tiles and WLS fibers. The estimated maximum total dose in the barrel hadronic calorimeter is of the order of $23 \mathrm{krad}$, and $36 \mathrm{krad}$ in the extended barrel [4]. 


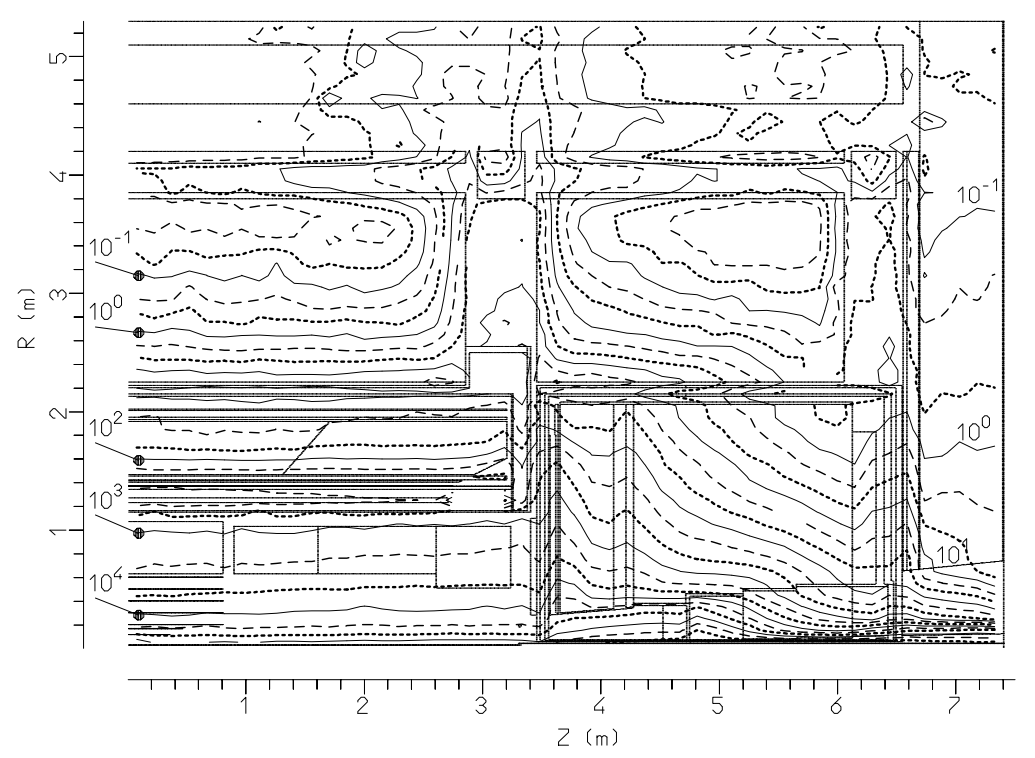

Figure 1: Yearly integrated dose $(G y /$ year $)$ in the inner detector and calorimeters.

\subsection{Low dose rate effects}

Some authors observed [5], that low dose rate effects exist in plastic scintillators: the damage of some scintillators and fibers submitted to a given total dose in a short time irradiation (high dose rate), is lower than the damage due to a long time irradiation (low dose rate), for the same total dose.

This matter has been a subject of large controversy. As an example, K. Wick and U. Holm [6], did not observe low dose rate effects in SCSN38 scintillator samples, while E. Biagtan et al. [7], observed low dose rate effects in several types of scintillator samples (including the SCSN38 scintillator). It should be stressed that in both experiments the geometry of the samples tested were similar, and the total dose and dose rate were of the same order of magnitude.

\subsection{Parametrization of irradiated fibers}

Experimental results for different types of scintillating and WLS plates and fibers, shows that both scintillation and transmission of light decreases with increasing dose. The following parametrization describes satisfactory the radiation damage of plastic 
scintillators and wavelengthshifters [8]:

$$
\frac{I_{\text {irr }}(x)}{I_{\text {notirr }}(x)}=\exp \left(-\frac{D(x)}{\delta}-\alpha \times \int_{0}^{x} D(s) d s\right)
$$

$D(x)$ is the total dose at a distance $x$ from the readout end of the fiber, $\delta$ is the parameter which characterizes the loss in emission, and $\alpha$ characterizes the loss in transmission. Radiation hard scintillators and WLS are characterized by high values of $\delta$ and low values of $\alpha$.

\section{Dose rate studies in WLS fibers irradiated with $\gamma$ 's}

\subsection{Dosimetry and simulation of dose profile}

A ${ }^{60} \mathrm{Co} \gamma$ source was used to irradiate the fibers, and dosimetry was made with Elcugray alanine cables. Fig. 2 shows the dose rate profile measured for the 3 irradiation positions, and also the results of a simulation with the EGS monte-carlo code [9], to predict these profiles. The dose profiles obtained are the following: a negligible dose rate between 0 and $50 \mathrm{~cm}(<200 \mathrm{rad} / \mathrm{h}$ for position $A$ and $<30 \mathrm{rad} / \mathrm{h}$ for position $B$ and $C$ ), a mean dose rate in the plateau from 50 to $200 \mathrm{~cm}$ of $3.9 \mathrm{krad} / \mathrm{h}$ for pos. $A$, $1.1 \mathrm{krad} / \mathrm{h}$ for pos. $B$ and $0.53 \mathrm{krad} / \mathrm{h}$ for pos. $C$.

\subsection{Radiation damage of WLS fibers}

The following types of WLS fibers, were irradiated [10]:

- Bicron: BCF99-28, BCF99-28 with 600 p.p.m. of UV absorber and BCF91A (all from a 1994 production).

- Kuraray: Y11(200)MS and Y11(200)MS with 1000 p.p.m. of UVA double cladding fibers (from a 1994 production).

- Pol.Hi.Tech: S048-100 N4 (1995 production) and S048-100 with UVA (1994 production, UVA concentration not specified).

All fibers have Polystyrene (PS) base, and PMMA cladding. Kuraray fibers have a second cladding of fluorinated PMMA. The fibers are $2 \mathrm{~m}$ long and $1 \mathrm{~mm}$ diameter, and they are polished at both ends. Five fibers of each type were used for each irradiation position.

Fig. 3 shows the light output $(I(x))$ for some typical fibers, and the fit of the experimental results to: 

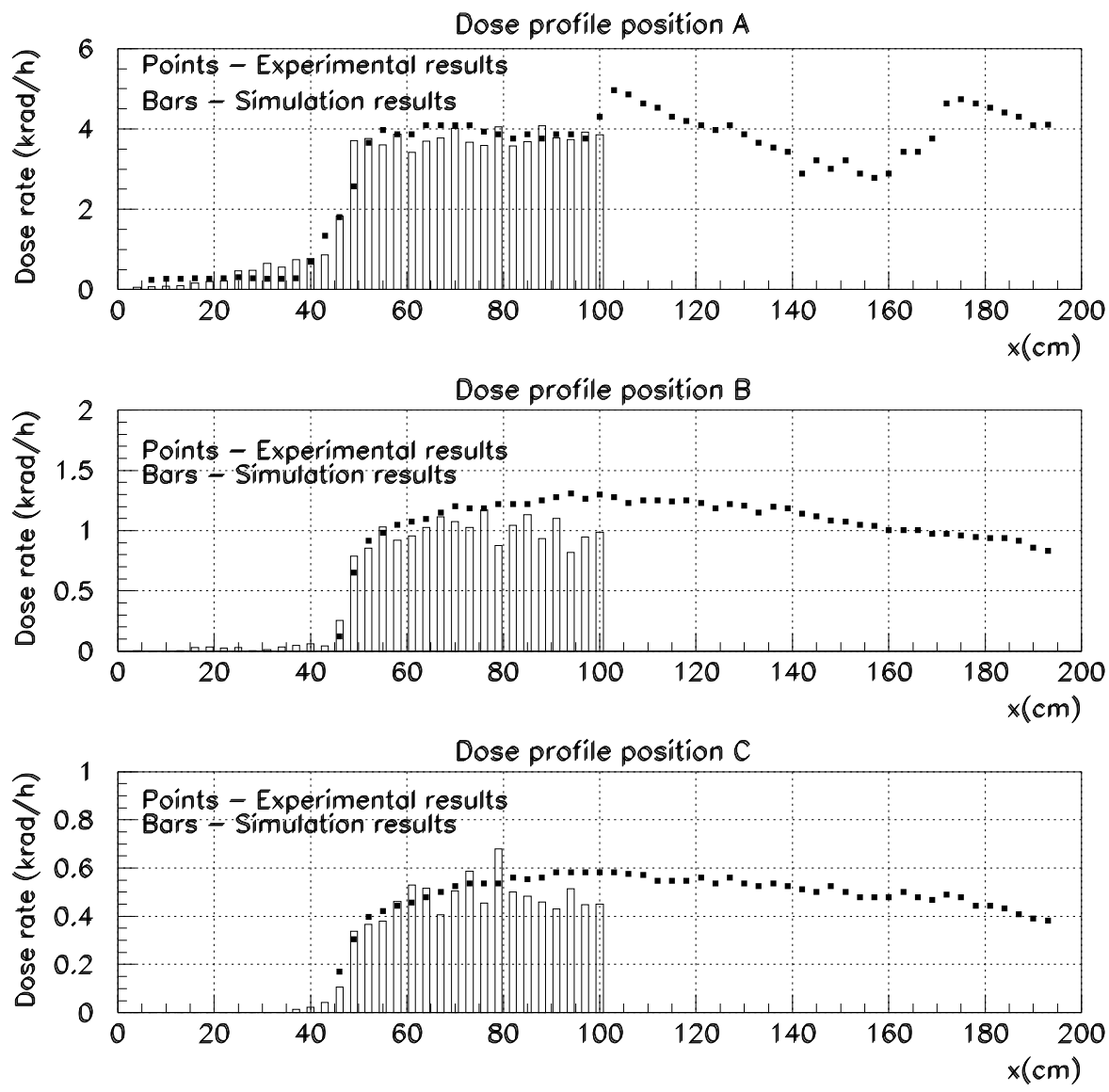

Figure 2: Dose profile for position A (top), B (middle) and C (bottom) - experimental and simulation results.

$$
I(x)=I_{0 S} \exp \left(-\frac{x}{L_{a t t S}}\right)+I_{0 L} \exp \left(-\frac{x}{L_{a t t L}}\right)
$$

$L_{\text {attS }}$ is the short attenuation length, $L_{a t t L}$ is the long attenuation length and $I_{0 T}=I_{0 S}+I_{0 L}$ is the light yield. The results of this fit are summarized in table 1.

All the fibers have a short attenuation length $\left(L_{\text {att } S}\right)$ ranging between $\sim 15$ and $20 \mathrm{~cm}$. The long attenuation length $\left(L_{\text {att } L}\right)$ is larger than $300 \mathrm{~cm}$ except for the Bicron BCF 99-28 fibers, which is $\sim 270 \mathrm{~cm}$. The BCF99-28 fibers with UVA have a $L_{\text {att } L}$ $25 \%$ lower than the corresponding fibers without UVA.

The light yield $\left(I_{0 T}\right)$ of the BCF99-28 fibers with and without UVA is similar, but the light output at $x=180 \mathrm{~cm}(I(180))$ is $19 \%$ lower for the fibers with UVA. Y11(200)MS fibers with UVA have $I_{0 T}$ and $I(180)$ higher by $\sim 6 \%$ than the same fibers without UVA. The fiber to fiber fluctuations are $\leq 3.5 \%$ in the light yield and 


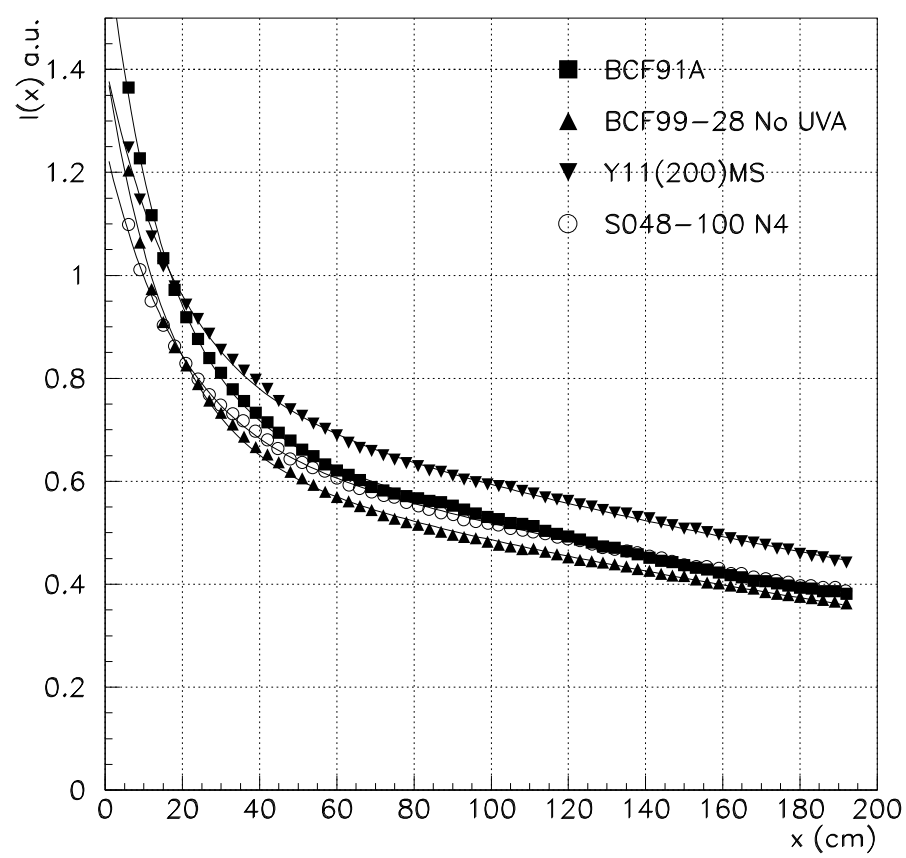

Figure 3: Light output for some typical fibers. The superimposed curves are the results from fitting the function of eq. 2 to the experimental data.

light output.

\subsection{Dose rate effects and recovery of the fibers}

The fibers were irradiated with an average total dose of $130 \mathrm{krad}$ for position $A$ and $140 \mathrm{krad}$ for positions $B$ and $C$. This corresponds to approximately 4 times the expected dose levels for the TILECAL at $\eta=1.5$, for 10 years of LHC operation.

Figure 4-left shows the following ratio: $R(x)=\frac{I_{i r r}(x)}{I_{n o t i r r}(x)}$, for the fibers without UVA, 2 months after the end of irradiation in position $\mathrm{C}(550 \mathrm{rad} / \mathrm{h})$. An error of $\pm 3 \%$ is estimated for $R(x)$ due to fiber to fiber fluctuations plus instrumental effects. The Y11(200)MS fibers show a light output loss of $11 \%$, at $x=180 \mathrm{~cm}$. The BCF 99-28 fibers have a $18 \%$ decrease, and the S048-100 N4 a $38 \%$ light output loss at $x=180 \mathrm{~cm}$. Similar results are obtained for the other 2 dose rates for all the fibers, except the BCF91A samples which show a $10 \%$ higher light output loss in the lower dose rate position $(0.55 \mathrm{krad} / \mathrm{h})$ than in the higher dose rate positions (see table 5 ). This can be due to some further damage in the fibers which was not controlled, to some global error in the measurement, or to some natural ageing after irradiation.

Tables 2 and 3 show the transmission loss $(\alpha)$ and the emission loss $(\delta)$ parameters, respectively. Within the fibers without UVA, the Y11(200)MS fibers are the ones with both the highest values of $\delta(5.3 \mathrm{Mrad})$ and the lowest values of $\alpha, 4.9 \times 10^{-3}$ $(\text { Mrad.cm })^{-1}$. 


\begin{tabular}{|c|c|c|c|c|}
\hline Fiber type & $L_{\text {attS }}(\mathrm{cm})$ & $L_{\text {attL }}(\mathrm{cm})$ & $I_{0 T}$ & $I(180)$ \\
\hline BCF99-28 No UVA & $14.6 \pm 1.0$ & $271 \pm 2$ & $1.674 \pm 0.039$ & $\mathbf{0 . 3 9 4} \pm \mathbf{0 . 0 1 2}$ \\
BCF99-28 600 ppm UVA & $15.4 \pm \mathbf{0 . 6}$ & $204 \pm 8$ & $1.673 \pm \mathbf{0 . 0 4 3}$ & $\mathbf{0 . 3 2 0} \pm \mathbf{0 . 0 0 9}$ \\
BCF91-A & $16.0 \pm \mathbf{0 . 7}$ & $308 \pm 10$ & $1.415 \pm \mathbf{0 . 0 4 5}$ & $\mathbf{0 . 3 7 6 \pm 0 . 0 0 6}$ \\
\hline Y11(200)MS & $18.4 \pm \mathbf{0 . 4}$ & $327 \pm \mathbf{5}$ & $1.410 \pm \mathbf{0 . 0 2 6}$ & $\mathbf{0 . 4 5 9} \pm \mathbf{0 . 0 1 5}$ \\
Y11(200)MS 1000 ppm UVA & $18.4 \pm \mathbf{0 . 8}$ & $313 \pm 17$ & $1.495 \pm \mathbf{0 . 0 5 9}$ & $\mathbf{0 . 4 8 2} \pm \mathbf{0 . 0 1 7}$ \\
\hline S048-100-N4 & $18.8 \pm 1.3$ & $\mathbf{3 2 9} \pm 17$ & $1.254 \pm \mathbf{0 . 0 4 4}$ & $\mathbf{0 . 3 9 8} \pm \mathbf{0 . 0 1 5}$ \\
S048-100 with UVA & $19.3 \pm 1.3$ & $333 \pm 20$ & $\mathbf{0 . 7 8 9} \pm \mathbf{0 . 0 1 8}$ & $\mathbf{0 . 2 4 3} \pm \mathbf{0 . 0 0 5}$ \\
\hline
\end{tabular}

Table 1: Values obtained for $L_{\text {att } S}$ - Short attenuation length, $L_{a t t L}$ - long attenuation length, $I_{0 T}$ - light yield, $I(180)$ - light output at $x=180 \mathrm{~cm}$. Mean and RMS of 5 fibers of each type.

For $\delta=5.3$ Mrad and a total dose of $140 \mathrm{krad}$ the computed emission loss is: $\sim 3 \%$. This means that from a total light output loss of $\sim 10 \%$ at $x \sim 200 \mathrm{~cm}$, about $7 \%$ is due to the transmission loss.

Figure 4-right shows $R(x)$ for the fibers with UVA. The fibers which lose less light are the Y11(200)MS with 1000 p.p.m.. of UVA, with $13 \%$ decrease at $x=180 \mathrm{~cm}$. The S048-100 fibers with UVA have a $22 \%$ light output loss, being slightly radiation harder than the BCF99-28 with 600 p.p.m. of UVA ( $26 \%$ of light loss).

Tables 4 and 5 summarize the values of $R(180)$ for all fiber types irradiated in the 3 positions, obtained 4 hours and 2 or 3 months after the end of irradiation, respectively. It can be seen that the addition of 1000 p.p.m. of UVA to the Y11(200)MS fibers, has a negligible effect on the radiation hardness of these fibers. The BCF99-28 fibers with 600 p.p.m. of UVA, lose $\sim 7 \%$ more light than the ones without UVA. We have not made a direct comparison between S048-100 N4 fibers and S048-100 UVA, since these fibers are not of the same type. S048-100 UVA fibers (1994 production) are radiation harder than the S048-100 N4 fibers (1995 production), which is consistent with the type of fibers used. Since shorter wavelengths are more sensitive to ionizing radiation, the UV absorber decreases the effect of radiation on the fibers [11].

Comparing tables 4 and 5 , it can be seen that all fibers recover part of the initial light output loss, except BCF91A fibers. These fibers present a higher damage at 2 months after irradiation, than at 4 hours. The reason for this behaviour is due to some uncontrolled error in the measurement or to some further damage in the fibers, as explained earlier.

Now we will look for low dose rate effects. This experiment was done in such a way that the total dose and the fiber types, are the same in the 3 irradiation positions. Care was taken so that the only parameter that changed was the dose rate.

The BCF99-28 fibers with and without UVA, measured 4 hours after the end 


\begin{tabular}{|c|c|c|c|c|}
\hline \multirow{2}{*}{ Fiber type } & \multicolumn{4}{|c|}{$\alpha \times 10^{3}\left(\mathrm{Mrad}_{\mathrm{cm}}\right)^{-1}$ Position C, Time after irrad. } \\
\cline { 2 - 5 } & $(4$ hours $)$ & $(1$ days $)$ & $(\mathbf{7}$ days $)$ & $(2$ months $)$ \\
\hline BCF99-28 No UVA & $12.1 \pm \mathbf{0 . 2}$ & $11.3 \pm 1.1$ & $11.6 \pm \mathbf{0 . 9}$ & $9.0 \pm \mathbf{0 . 7}$ \\
BCF99-28 600 ppm UVA & $15.3 \pm \mathbf{0 . 6}$ & $14.7 \pm 1.0$ & $15.5 \pm \mathbf{0 . 6}$ & $13.0 \pm 1.3$ \\
BCF91-A & $10.6 \pm 0.7$ & $10.3 \pm \mathbf{0 . 5}$ & $12.6 \pm \mathbf{0 . 3}$ & $12.2 \pm \mathbf{0 . 5}$ \\
\hline Y11(200)MS & $6.7 \pm \mathbf{0 . 4}$ & $6.6 \pm 0.5$ & $6.0 \pm 0.4$ & $4.9 \pm \mathbf{0 . 3}$ \\
Y11(200)MS 1000 ppm UVA & $8.1 \pm \mathbf{0 . 4}$ & $7.6 \pm \mathbf{0 . 4}$ & $7.2 \pm \mathbf{0 . 5}$ & $5.8 \pm \mathbf{0 . 4}$ \\
\hline S048-100-4 & $27.7 \pm 0.8$ & $26.5 \pm \mathbf{0 . 4}$ & $26.0 \pm \mathbf{0 . 8}$ & $21.4 \pm \mathbf{0 . 7}$ \\
S048-100 with UVA & $16.9 \pm 0.9$ & $15.8 \pm \mathbf{0 . 5}$ & $15.4 \pm \mathbf{0 . 6}$ & $12.4 \pm \mathbf{0 . 5}$ \\
\hline
\end{tabular}

Table 2: WLS fibers irradiated in position C $(550 \mathrm{rad} / \mathrm{h})$. Each column shows the value of the transmission loss $\alpha$, after the end of irradiation. Mean and RMS for 5 fibers of each type.

of irradiation (table 4), show a loss in the light output which is similar for the 3 dose rates. The light output loss of the Y11(200)MS fibers slightly decreases with decreasing dose rate. This difference is negligible for the fibers without UVA and $\simeq 7 \%$ for the fibers with UVA. A much higher effect is seen in both S048-100 fibers. The light loss in dose rate $\mathrm{A}$ is $15 \%$ higher than in dose rate $\mathrm{C}$ for the S048-100 N4 fibers, and $11 \%$ for the S048-100 UVA fibers.

After 2-3 months of recovery (table 5), the BCF99-28 and Y11(200)MS fibers present a similar light loss in the 3 dose rate positions. The S048-100 fibers with UVA present a similar damage in both dose rates $A$ and $C$, but the S048-100 N4 fibers show less damage at the lower dose rate $\mathrm{C}$ than at the higher dose rate $\mathrm{A}$ (difference of $6 \%$ ).

The decrease in the light output loss with increasing dose rate, can be explained by the competition of two processes: The deterioration by ionizing radiation and the recovery by annealing and diffusion of oxygen into the fiber, see K. Wick in [6]. In this way, after the end of a long term (low dose rate) irradiation, the fibers can present a lower damage due to a longer recovery time, than at the end of a fast term (high dose rate) irradiation. This can explain why after 2-3 months of recovery, a similar damage for the 3 dose rates is observed, or at least a smaller difference (as is the case of S048-100 N4 fibers).

In very long term irradiations the damage due to the natural ageing of the fibers can be of the same order of the damage caused by ionizing radiation, masking this effect. 


\begin{tabular}{|c|c|c|c|c|}
\hline \multirow{2}{*}{ Fiber type } & \multicolumn{4}{|c|}{$\delta$ (Mrad) Position C, Time after irrad. } \\
\cline { 2 - 5 } & $(4$ hours $)$ & (1 days) & (7 days) & (2 months) \\
\hline BCF99-28 No UVA & $3.4 \pm 0.9$ & $2.9 \pm \mathbf{0 . 2}$ & $1.9 \pm 0.3$ & $4.1 \pm 1.7$ \\
BCF99-28 600 ppm UVA & $3.2 \pm 1.3$ & $2.4 \pm \mathbf{0 . 9}$ & $1.8 \pm \mathbf{0 . 7}$ & $3.1 \pm 1.1$ \\
BCF91-A & $2.7 \pm 0.9$ & $2.9 \pm \mathbf{0 . 4}$ & $1.2 \pm 0.1$ & $1.7 \pm \mathbf{0 . 2}$ \\
\hline Y11(200)MS & $5.2 \pm 1.1$ & $6.5 \pm 1.0$ & $3.8 \pm 0.6$ & $5.3 \pm 0.8$ \\
Y11(200)MS 1000 ppm UVA & $4.4 \pm 0.7$ & $5.5 \pm 1.1$ & $3.7 \pm 0.5$ & $5.5 \pm 1.1$ \\
\hline S048-100-4 & $1.3 \pm 0.2$ & $1.6 \pm \mathbf{0 . 3}$ & $1.2 \pm 0.1$ & $1.9 \pm \mathbf{0 . 3}$ \\
S048-100 with UVA & $5.5 \pm 2.0$ & $5.8 \pm 2.4$ & $3.1 \pm 0.9$ & $5.9 \pm 3.9$ \\
\hline
\end{tabular}

Table 3: WLS fibers irradiated in position C (550 rad/h). Each column shows the value of the emission loss $\delta$, after the end of irradiation. Mean and RMS for 5 fibers of each type.

\section{Radiation damage of WLS fibers irradiated with}

$$
\text { neutrons }+\gamma^{\prime} \mathbf{s}
$$

Bicron BCF91A (1995 production), and Kuraray Y11(200) fibers (1993 production) both with single and double cladding, were irradiated in the mixed field $(80 \% \gamma$ 's + $20 \%$ neutrons) of a nuclear reactor. Five $150 \mathrm{~cm}$ long fibers of each type were tested.

A 'peak' total dose of $1 \mathrm{Mrad}$ at a dose rate of about $1.5 \mathrm{Mrad} / \mathrm{h}$ was applied. An estimate of the dose profile is given in fig. 5 [12].

Table 6 summarizes the values obtained for the ratio $R(x)$ at $x=130 \mathrm{~cm}$, corresponding to the peak of the dose profile. The recovery process was monitored between 1 hour and 20 days after the end of the irradiation. Fig. 6-left shows the ratio of light output after irradiation over light output before irradiation, for the fibers $\mathrm{Y} 11(200)$, Y11(200)M and BCF91A measured 1 hour after the end of irradiation, and fig. 6-right the recovery of $\mathrm{Y} 11(200) \mathrm{M}$ fibers.

It can be seen that the initial damage is very strong (of the order of 70-80\%). The fibers recover in about 6 days to the permanent damage, since no difference was observed between 6 days and 20 days after the end of irradiation.

The permanent damage is $18 \%$ for the $\mathrm{Y} 11(200)$ fibers (single and double cladding), and somewhat smaller for the BCF91A fibers (11\% for double cladding and $14 \%$ for single cladding).

Experimental values obtained at high dose rate (table 6 ), are compared with values computed using $\alpha$ and $\delta$ parameters from low dose rate experiments (tables 2 and 3 ). Table 7 shows the values of $R(130)$ obtained experimentally in the second column, and obtained with the extrapolation from the lower dose irradiation (third column). As can be seen, the experimental and calculated values of this table have a negligible 

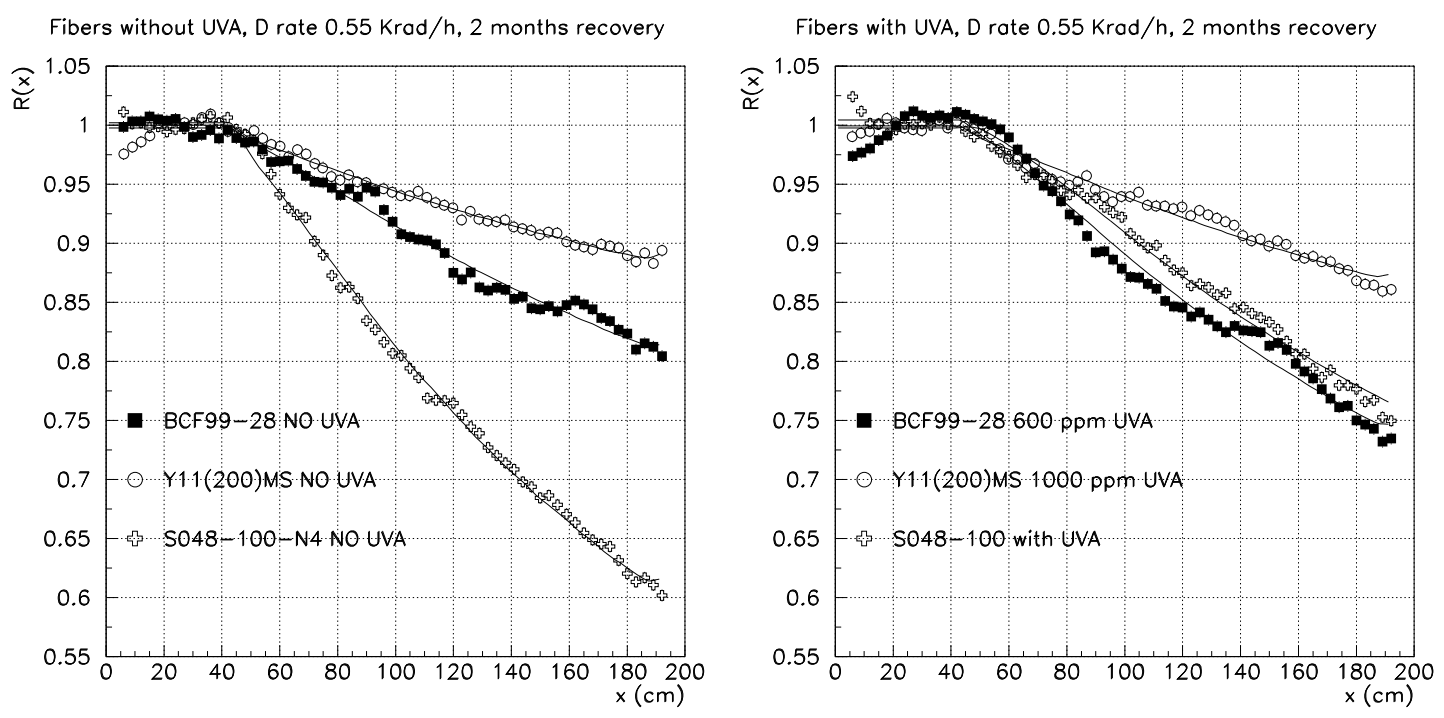

Figure 4: Light output after irradiation over light output before irradiation, for the fibers without UVA (left), and with UVA (right). Fibers irradiated in position C $(550 \mathrm{rad} / \mathrm{h})$, measured 2 month after the end of irradiation.

difference, no low dose rate effects are observed, neither effects from the neutron component of the irradiation field. This is consistent with results from other authors $[8][13]$.

\section{Conclusions}

Concerning the ${ }^{60} \mathrm{Co} \gamma$ irradiation we conclude that the Kuraray fibers are radiation harder than the Bicron and Pol.Hi.Tech fibers. The UV absorber used by Kuraray has no effect neither in the optical properties nor in the sensitivity to ionizing radiation. On the other hand the UVA used by Bicron, has the effect of lowering the attenuation length and making the fibers less resistant to radiation.

We also conclude that no low dose rate effects are observed for the Polystyrene base WLS fibers studied, with a total dose of 130-140 krad and at dose rates ranging between $550 \mathrm{rad} / \mathrm{h}$ and $4 \mathrm{krad} / \mathrm{h}$. It should be emphasized that the maximum expected dose rate in the TILECAL calorimeter is $\sim 1 \mathrm{rad} / \mathrm{h}$, which is $2-3$ orders of magnitude lower than the dose rates studied in this work.

In respect to the neutrons $+\gamma$ irradiation we conclude that for a total dose of $1 \mathrm{Mrad}$ at a very high dose rate $(1.5 \mathrm{Mrad} / \mathrm{h})$, single and multicladding fibers from the same producer, have similar light output loss. Y11(200) fibers are slightly less 


\begin{tabular}{|c|c|c|c|}
\hline \multirow{2}{*}{ Fiber type } & \multicolumn{3}{|c|}{$R(180) 4$ hours after irrad. } \\
\cline { 2 - 4 } & $\mathrm{A}(4 \mathrm{krad} / \mathrm{h})$ & $\mathrm{B}(1.1 \mathrm{krad} / \mathrm{h})$ & $\mathrm{C}(550 \mathrm{rad} / \mathrm{h})$ \\
\hline BCF99-28 No UVA & $\mathbf{0 . 7 8} \pm \mathbf{0 . 0 2}$ & $\mathbf{0 . 7 4} \pm \mathbf{0 . 0 2}$ & $\mathbf{0 . 7 8} \pm \mathbf{0 . 0 1}$ \\
BCF99-28 600 ppm UVA & $\mathbf{0 . 7 2} \pm \mathbf{0 . 0 1}$ & $\mathbf{0 . 6 7} \mathbf{0 . 0 1}$ & $\mathbf{0 . 7 2} \pm \mathbf{0 . 0 2}$ \\
BCF91-A & $\mathbf{0 . 8 4} \pm \mathbf{0 . 0 2}$ & $\mathbf{0 . 7 6} \pm \mathbf{0 . 0 1}$ & $\mathbf{0 . 7 9} \pm \mathbf{0 . 0 2}$ \\
\hline Y11(200)MS & $\mathbf{0 . 8 2} \pm \mathbf{0 . 0 1}$ & $\mathbf{0 . 8 3} \pm \mathbf{0 . 0 1}$ & $\mathbf{0 . 8 6} \pm \mathbf{0 . 0 2}$ \\
Y11(200)MS 1000 ppm UVA & $\mathbf{0 . 7 7} \pm \mathbf{0 . 0 1}$ & $\mathbf{0 . 8 1} \pm \mathbf{0 . 0 1}$ & $\mathbf{0 . 8 4} \pm \mathbf{0 . 0 1}$ \\
\hline S048-100-4 & $\mathbf{0 . 3 9} \pm \mathbf{0 . 0 1}$ & - & $\mathbf{0 . 5 4} \pm \mathbf{0 . 0 1}$ \\
S048-100 with UVA & $\mathbf{0 . 5 8} \pm \mathbf{0 . 0 1}$ & - & $\mathbf{0 . 7 1} \pm \mathbf{0 . 0 1}$ \\
\hline
\end{tabular}

Table 4: Ratio $R(180)$. Fibers measured 4 hours after the end of irradiation. Mean and RMS of 5 fibers of each type.

\begin{tabular}{|c|c|c|c|}
\hline \multirow{2}{*}{ Fiber type } & \multicolumn{3}{|c|}{$R(180) 2-3$ month after irrad. } \\
\cline { 2 - 4 } & $\mathrm{A}(4 \mathrm{krad} / \mathrm{h})$ & $\mathrm{B}(1.1 \mathrm{krad} / \mathrm{h})$ & $\mathrm{C}(550 \mathrm{rad} / \mathrm{h})$ \\
\hline BCF99-28 No UVA & $\mathbf{0 . 8 6} \pm \mathbf{0 . 0 2}$ & $\mathbf{0 . 8 5} \pm \mathbf{0 . 0 2}$ & $\mathbf{0 . 8 2} \pm \mathbf{0 . 0 2}$ \\
BCF99-28 600 ppm UVA & $\mathbf{0 . 7 9} \pm \mathbf{0 . 0 2}$ & $\mathbf{0 . 7 7} \pm \mathbf{0 . 0 3}$ & $\mathbf{0 . 7 4} \pm \mathbf{0 . 0 2}$ \\
BCF91-A & $\mathbf{0 . 8 1 \pm \mathbf { 0 . 0 1 }}$ & $\mathbf{0 . 8 3} \pm \mathbf{0 . 0 2}$ & $\mathbf{0 . 7 4} \pm \mathbf{0 . 0 1}$ \\
\hline Y11(200)MS & $\mathbf{0 . 9 0} \pm \mathbf{0 . 0 1}$ & $\mathbf{0 . 9 3} \pm \mathbf{0 . 0 2}$ & $\mathbf{0 . 8 9} \pm \mathbf{0 . 0 1}$ \\
Y11(200)MS 1000 ppm UVA & $\mathbf{0 . 8 7} \pm \mathbf{0 . 0 2}$ & $\mathbf{0 . 9 0} \pm \mathbf{0 . 0 1}$ & $\mathbf{0 . 8 7} \pm \mathbf{0 . 0 1}$ \\
\hline S048-100-4 & $\mathbf{0 . 5 7} \pm \mathbf{0 . 0 1}$ & - & $\mathbf{0 . 6 2} \pm \mathbf{0 . 0 2}$ \\
S048-100 with UVA & $\mathbf{0 . 7 7} \pm \mathbf{0 . 0 2}$ & - & $\mathbf{0 . 7 8} \pm \mathbf{0 . 0 2}$ \\
\hline
\end{tabular}

Table 5: Ratio $R(180)$. Fibers measured 2-3 month after the end of irradiation. Mean and RMS of 5 fibers of each type.

resistant than BCF91A fibers. The Kuraray fibers used in this study are from an older production (1993), this can explain the contradiction with the ${ }^{60} \mathrm{Co}$ irradiation. No low dose rate effects were observed when compared with the lower dose rate irradiation, neither effects due to the neutron component of the irradiation field.

\section{Acknowledgments}

We thank Dr. E. Andrade from ITN Sacavém for providing the ${ }^{60}$ Co irradiation facility. To Dr. E. Martinho and Eng. A. Craveira from the RPI Sacavém for helping in the irradiation of the fibers in the nuclear reactor. We thank to U. Holm by helpful discussions about those results. This work was supported by JNICT Portugal. 


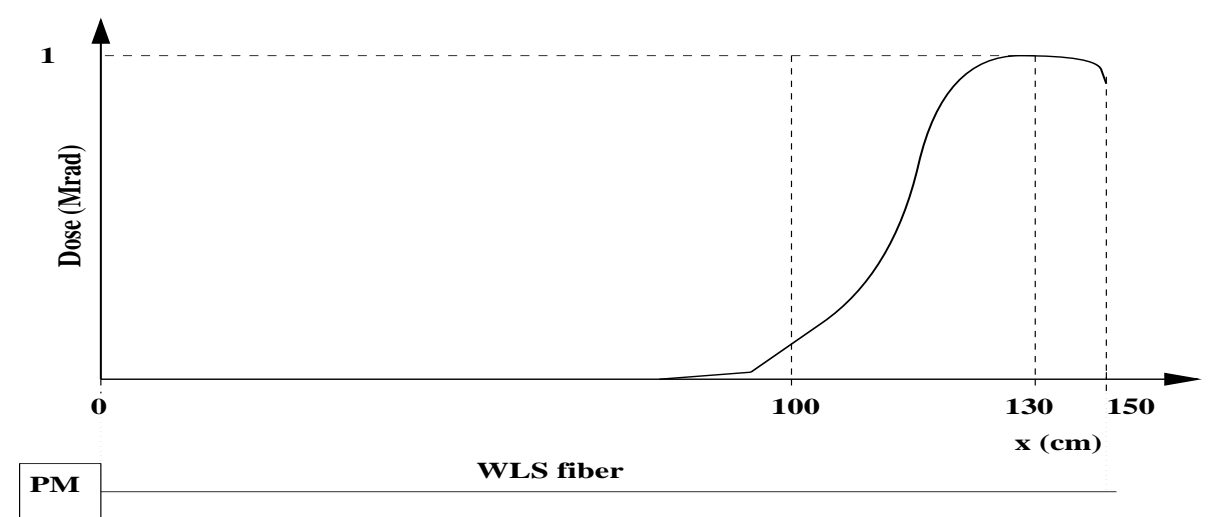

Figure 5: Estimated dose profile in the nuclear reactor.

\begin{tabular}{|c|c|c|c|c|c|}
\hline \multirow{2}{*}{ Fiber type } & \multicolumn{5}{|c|}{$R(130) \pm R M S(\%)$, time after irrad. } \\
\cline { 2 - 6 } & $(1$ hours $)$ & $(3$ hours $)$ & $(20$ hours $)$ & $(6$ days $)$ & $(20$ days $)$ \\
\hline BCF91-A & $\mathbf{0 . 2 6} \pm 2.8 \%$ & $0.30 \pm 3.2 \%$ & - & $\mathbf{0 . 7 6} \pm \mathbf{0 . 7 \%}$ & $\mathbf{0 . 7 9} \pm \mathbf{0 . 7 \%}$ \\
BCF91-A DC & $\mathbf{0 . 3 7} \pm 3.5 \%$ & $0.40 \pm 3.9 \%$ & - & $\mathbf{0 . 7 6} \pm 2.0 \%$ & $\mathbf{0 . 7 6} \pm 1.7 \%$ \\
\hline Y11(200) & $\mathbf{0 . 2 0} \pm 2.8 \%$ & $\mathbf{0 . 2 2} \pm 2.8 \%$ & $\mathbf{0 . 6 0} \pm 2.8 \%$ & $\mathbf{0 . 7 0} \pm 1.2 \%$ & $\mathbf{0 . 7 2} \pm \mathbf{3 . 7 \%}$ \\
Y11(200)M & $\mathbf{0 . 1 9} \pm 1.6 \%$ & $\mathbf{0 . 2 2} \pm 2.1 \%$ & $\mathbf{0 . 6 4} \pm 1.8 \%$ & $\mathbf{0 . 7 0} \pm 1.0 \%$ & $\mathbf{0 . 7 2} \pm \mathbf{2 . 8 \%}$ \\
\hline
\end{tabular}

Table 6: Ratio $R(130)$. WLS fibers irradiated with neutron $+\gamma$ 's. Mean and RMS of 5 fibers of each type.

\section{References}

[1] ATLAS Collaboration, ATLAS, Technical Proposal for a General-Purpose pp Experiment at the Large Hadron Collider at CERN. CERN/LHCC/94-43 LHCC/P2, 15 December 1994

[2] F. Ariztizabal et al., Nucl. Instrum. Methods A349 (1994) 384

[3] G. Battistoni et al., ATLAS Internal Note GEN-No-010, 1994

[4] A. Amorim, M. David, A. Gomes, A. Henriques, A. Maio "Study of the effect of the radiation on the TILECAL Barrel hadron calorimeter to be used in ATLAS." Presented by A. Henriques, "IV International Conference on calorimetry in High Energy Physics", La Biodola, Elba, Italy September 1993, pp. 669-673 

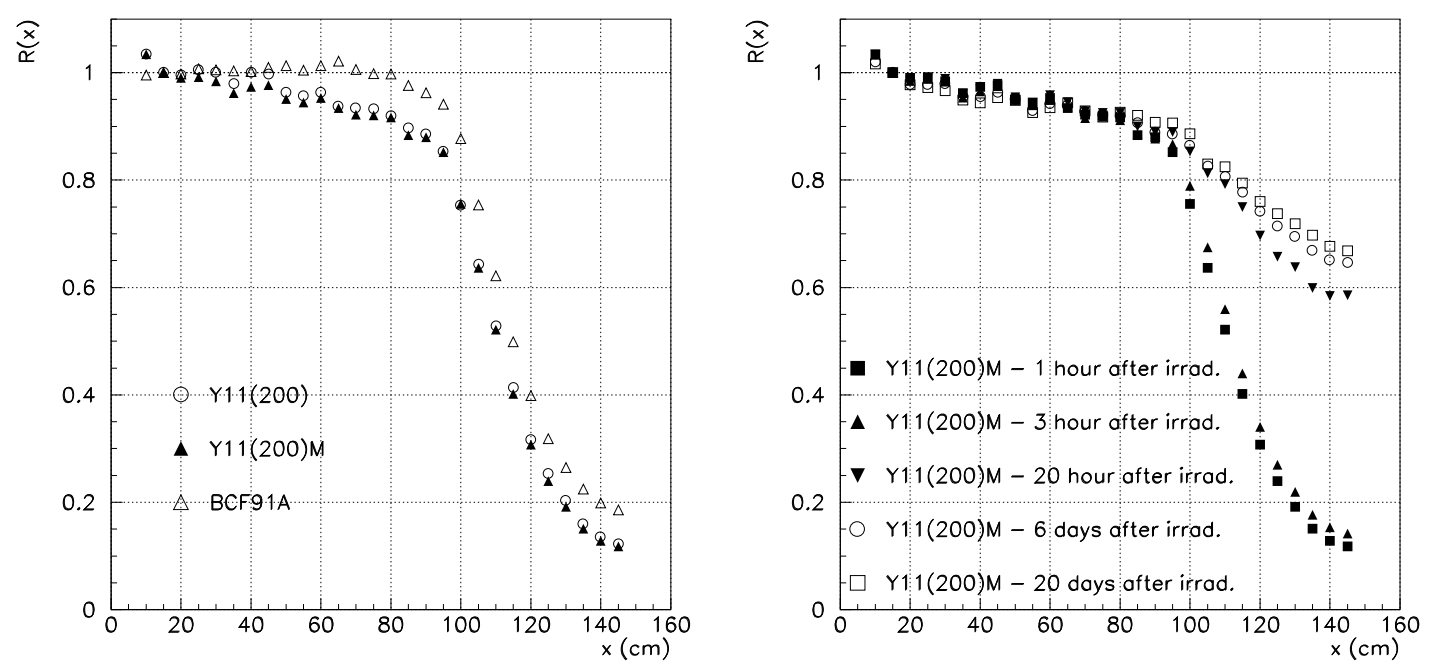

Figure 6: Light output after irradiation over light output before irradiation, for the fibers irradiated with neutrons. First measurement (1 hour) after the end of irradiation (left), fibers Y11(200), Y11(200)M and BCF91A. Recovery of Y11(200)M fibers (right).

[5] Y. Sirois and R. Wigmans, Nucl. Instrum. Methods A240 (1985) 262

[6] K. Wick et al., Nucl. Instrum. Methods B61 (1991) 472

[7] E.Biagtan et al., Nucl. Instrum. Methods B93 (1994) 296

[8] A. Maio, Large Hadron Collider Workshop, vol. III, Aachen, October 1990, pp 625. CERN 90-10, ECFA 90-133, 1990

[9] W.R. Nelson, H. Hirayama, D.W.O. Rogers, The EGS4 Code System, SLAC-265 (1985).

[10] M. David Master Thesis, Faculdade de Ciências da Universidade de Lisboa, 1996

[11] Dr. Meoni from Pol.Hi.Tech, private communication.

[12] E. Martinho, "Calculation of total absorbed doses in research reactors", Kernitechnik 61 (1996) 2-3, pp 122, and private communications 1996

[13] G. Bub et al., IEEE Trans. Nuc. Sci. vol. 42, No. 4, 1995, 315 


\begin{tabular}{|c|c|c|}
\hline \multirow{2}{*}{ Fiber type } & \multicolumn{2}{|c|}{$R(130) \pm R M S(\%)$} \\
\cline { 2 - 3 } & Experimental (high dose rate) & Calculated from low dose rate results \\
\hline BCF91-A & $\mathbf{0 . 7 9} \pm \mathbf{0 . 7 \%}$ & $\mathbf{0 . 7 3}$ \\
Y11(200)M & $0.72 \pm 2.8 \%$ & $\mathbf{0 . 7 5}$ \\
\hline
\end{tabular}

Table 7: Ratio $R(130)$ obtained experimentally (second column), and calculated with $\alpha$ and $\delta$ from the lower dose irradiation (third column). 